\title{
Strange Star Equations of State Revisited
}

\author{
Debora P. Menezes ${ }^{\mathrm{A}, \mathrm{B}, \mathrm{C}}$ and Don B. Melrose ${ }^{\mathrm{B}}$ \\ A Depto de Física - CFM - CP476, Universidade Federal de Santa Catarina, Florianópolis - SC, Brazil \\ B School of Physics, University of Sydney, Sydney NSW 2006, Australia \\ C Corresponding author. Email: debora@physics.usyd.edu.au,debora@fsc.ufsc.br
}

Received 2005 June 8, accepted 2005 September 8

\begin{abstract}
Motivated by recent suggestions that strange stars can be responsible for glitches and other observational features of pulsars, we review some possible equations of state and their implications for models of neutron, hybrid, and strange stars. We consider the MIT bag model and also strange matter in the colourflavour locked phase. The central energy densities for strange stars are higher than the central densities of ordinary neutron stars. Strange stars are bound by the strong force and so can also rotate much faster than neutron stars. These results are only weakly dependent on the model used for the quark matter. If just one of the existing mass-to-radius ratio constraint is valid, most neutron stars equations of state are ruled out, but all the strange stars equations of state presented in this work remain consistent with the constraint.
\end{abstract}

Keywords: stars: interiors - equation of state

\section{Introduction}

Qualitatively, a neutron star is analogous to a white dwarf star, with the pressure due to degenerate neutrons rather than degenerate electrons. General relativity is significant for neutron stars, and the simplest models are found by solving the Tolman-Oppenheimer-Volkoff equations (Tolman 1939; Oppenheimer \& Volkoff 1939), which are derived from Einstein's equations in the Schwarzschild metric for a static, spherical star composed of an ideal gas. The assumption that the neutrons in a neutron star can be treated as an ideal gas is not well justified: The effect of the strong force needs to be taken into account by replacing the equation of state (EOS) for an ideal gas by a more realistic EOS. Neutron stars can be regarded as laboratories for testing different hypotheses relating to the EOS through their influence on the properties of neutron star models, including radius, mass, central density, and Kepler frequency. The effect of many possible EOS has been studied in this context (Glendenning 2000; Prakash et al. 1997). One class of EOS is based on the assumption that the neutron star is composed only of hadrons (Espíndola \& Menezes 2002; Santos \& Menezes 2004) plus an essential small admixture of electrons. In order to construct appropriate EOS for these hadronic stars, one must rely on models which describe nuclear matter's bulk properties. Another possibility is that the interior of the neutron star includes quarks; such models are known as quark stars (Ivanenko \& Kurdgelaidze 1969) or hybrid stars (Glendenning 2000). In hybrid stars low-density regions are composed of hadronic matter, but in high-density regions a deconfinement of the quarks from the hadrons occurs, leading to a quark phase. Many different EOS have been built, including EOS for the phase immediately after the formation of the neutron star when the neutrinos are still trapped (Panda et al. 2004a; Menezes \& Providência 2004a) and for the subsequent (deleptonized) phase after the neutrinos escape (Menezes \& Providência 2003, 2004b, 2004c; Panda et al. 2004b).

It is possible that the interior of a neutron-like star does not consist primarily of neutrons, but rather of the strange matter. Strange matter is composed of deconfined quarks, including up, down, and strange quarks, plus the leptons necessary to ensure charge neutrality (Bodmer 1971; Witten 1984). This possibility arises because at the high densities present in the interior of neutron stars, a phase transition from hadronic to quark phase is possible (Glendenning 2000; Menezes \& Providência 2003, $2004 b, 2004 c$ ). Approximately one-third of the quarks are strange quarks, with the exact fraction depending on the model used to describe the quark phase. For example, the strangeness content is slightly lower for the Nambu-JonaLasinio model (Nambu \& Jona-Lasinio 1961a, 1961b) than for the MIT bag model (Chodos et al. 1974), as can be seen in Menezes \& Providência (2003). It has been argued (Alcock et al. 1986) that strange matter is the true ground state of all matter, and if this is the case then as soon as the core of the star converts to the quark phase, the entire star converts. This led to the suggestion that there may be no neutron stars, and that all neutron-like stars are in fact strange stars (Alcock et al. 1986).

Further evidence in favour of strange stars is the fact that some stars seem to rotate faster that what would be expected for a neutron star (Glendenning 2000; Glendenning \& Weber 1992). Neutron stars are gravitationally bound with the pressure approaching zero as the density approaches zero. Strange stars, on the other hand, are self-bound by the strong interaction and gravity is not necessary for their stability. In this case the pressure goes 
to zero at a finite density, so that there is a sharp change in density at the surface of a strange star. The restriction on the rotational speed of a gravitationally bound star, such that it does not break up due to the centrifugal acceleration exceeding the gravitational acceleration at the equator of the star, might not apply to a strange star and, in this case, strange stars could rotate faster than neutron stars. It is however worth emphasizing that, although there are predictions that neutron or strange stars can rotate as fast as $1.5 \mathrm{kHz}$, such rapidly rotating objects have never been observed.

A strange star must have a thin layer on its surface dominated by the electrons, which are necessary to enforce charge neutrality. This layer could suspend a hadronic crust, which would not be in contact with the stellar core (Alcock et al. 1986; Glendenning \& Weber 1992). It has been suggested that the presence of a crust provides a natural explanation for pulsar glitches (Glendenning \& Weber 1992), which are sudden changes in the rotation period of the pulsar. Other authors have argued against the presence of a crust (Xu 2003a; Usov 1998, 2001) and that strange stars should be bare. Recently, Aksenov et al. (2004) identified some possible characteristics of the radiation that would come from hot, bare-strange stars. Although our main interest is in bare-strange stars, we investigate the extent to which the stellar properties are influenced by a hadronic crust.

In this paper we focus on the different possible models for strange matter and their implications for models of strange stars. Besides the MIT bag model (Chodos et al. 1974), normally used for strange stars, more sophisticated models are quark models with a colourflavour locked phase (CFL; Shovkovy et al. 2003; Son \& Stephanov 2000a, 200b; Buballa \& Oertel 2002; Alford et al. 2001; Alford \& Reddy 2003) and the Nambu-JonaLasinio model (Nambu \& Jona-Lasinio 1961a, 1961b). We consider only the MIT and the CFL phase models here. We also discuss the effects of the inclusion of a crust, not only in strange stars, for which the crust is not in contact with the interior, but also for hadronic and hybrid stars for which it is in contact with the interior. We calculate the Kepler frequencies using a prescription given in Lattimer \& Prakash (2004) to test the hypothesis that strange stars can rotate faster than neutron stars. Finally, we also investigate how changes in the EOS affect the star properties within the temperature range of $10-15 \mathrm{MeV}$.

\section{Quark Matter}

In this section we summarize the models we use to describe the properties of quark matter.

\subsection{MIT Bag Model}

The MIT bag model (Chodos et al. 1974) has been used extensively to describe quark matter. In its simplest form, the quarks are considered to be free inside a bag and the thermodynamic properties are derived by treating them as an ideal Fermi gas. The energy density, the pressure, and the quark q density, respectively, are given by

$$
\begin{gathered}
\varepsilon=3 \times 2 \sum_{\mathrm{q}=\mathrm{u}, \mathrm{d}, \mathrm{s}} \int \frac{\mathrm{d}^{3} p}{(2 \pi)^{3}} \sqrt{\mathbf{p}^{2}+m_{\mathrm{q}}^{2}}\left(f_{\mathrm{q}+}+f_{\mathrm{q}-}\right)+\text { Bag } \\
P=\frac{1}{\pi^{2}} \sum_{\mathrm{q}} \int \mathrm{d} p \frac{\mathbf{p}^{4}}{\sqrt{\mathbf{p}^{2}+m_{\mathrm{q}}^{2}}}\left(f_{\mathrm{q}+}+f_{\mathrm{q}-}\right)-\mathrm{Bag} \\
\rho_{\mathrm{q}}=3 \times 2 \int \frac{\mathrm{d}^{3} p}{(2 \pi)^{3}}\left(f_{\mathrm{q}+}-f_{\mathrm{q}-}\right)
\end{gathered}
$$

where 3 stands for the number of colours, 2 for the spin degeneracy, $m_{\mathrm{q}}$ for the quark masses, and Bag represents the bag pressure. The distribution functions for the quarks and anti-quarks are the Fermi-Dirac distributions

$$
f_{\mathrm{q} \pm}=1 /\left(1+\exp \left[\left(\epsilon \mp \mu_{\mathrm{q}}\right) / T\right]\right),
$$

with $\mu_{\mathrm{q}}$ the chemical potential for quarks (upper sign) and anti-quarks (lower sign) of type $q$ and energy $\epsilon=\sqrt{\mathbf{p}^{2}+m_{\mathrm{q}}^{2}}$. In our calculations we assume the masses $m_{\mathrm{u}}=m_{\mathrm{d}}=5.0 \mathrm{MeV}, m_{\mathrm{s}}=150.0 \mathrm{MeV}$, and adopt different values for the bag constant, Bag.

The foregoing equations apply at arbitrary temperature. For $T=0$, there are no antiparticles, the chemical potential is equal to the Fermi energy, and the distribution functions for the particles are the usual step functions $f_{\mathrm{q}+}=\theta\left(P_{\mathrm{Fq}}^{2}-p^{2}\right)$. For neutron stars, the typical temperatures are somewhat lower than the Fermi temperatures, and the approximation $T=0$ is normally made.

\subsection{Quark Matter in $\beta$-Equilibrium}

In a star with quark matter, we must impose both charge neutrality and equilibrium for the weak interactions, referred to as $\beta$-equilibrium (Glendenning 2000). In this work we only consider the stage after deleptonization when the neutrinos have escaped. In this case the neutrino chemical potential is zero. For matter in $\beta$-equilibrium, we must add the contribution of the leptons as free Fermi gases (electrons and muons) to the energy and pressure. The relations between the chemical potentials of the different particles are given by the $\beta$-equilibrium conditions

$$
\mu_{\mathrm{s}}=\mu_{\mathrm{d}}=\mu_{\mathrm{u}}+\mu_{\mathrm{e}} \quad \mu_{\mathrm{e}}=\mu_{\mu}
$$

For charge neutrality, we must impose

$$
\rho_{\mathrm{e}}+\rho_{\mu}=\frac{1}{3}\left(2 \rho_{\mathrm{u}}-\rho_{\mathrm{d}}-\rho_{\mathrm{s}}\right)
$$

For the electron and muon densities we have

$$
\rho_{1}=2 \int \frac{\mathrm{d}^{3} p}{(2 \pi)^{3}}\left(f_{1+}-f_{1-}\right) \quad 1=\mathrm{e}, \mu
$$

where the distribution functions for the leptons are given by substituting q by 1 in Equation (4), with $\mu_{1}$ the chemical potential for leptons of type 1. At $T=0$, Equation (6) becomes

$$
\rho_{1}=k_{\mathrm{Fl}}^{3} / 3 \pi^{2} .
$$


The pressure for the leptons is

$$
P_{1}=\frac{1}{3 \pi^{2}} \sum_{1} \int \frac{\mathbf{p}^{4} \mathrm{~d} p}{\sqrt{\mathbf{p}^{2}+m_{1}^{2}}}\left(f_{1+}+f_{1-}\right) .
$$

\subsection{Colour-Flavour Locked Quark Phase}

Recently many authors (Shovkovy et al. 2003; Son \& Stephanov 2000a, 2000b; Buballa \& Oertel 2002; Alford et al. 2001) have discussed the possibility that the quark matter is in a colour-superconducting phase, in which quarks near the Fermi surface are paired, forming Cooper pairs which condense and break the colour gauge symmetry (Alford 2001). At sufficiently high density the favoured phase is called CFL, in which quarks of all three colours and all three flavours are allowed to pair.

In this section, we study the equation of state taking into consideration a CFL quark-paired phase. We treat the quark matter as a Fermi sea of free quarks with an additional contribution to the pressure arising from the formation of the CFL condensates.

The CFL phase can be described with the thermodynamic potential (Alford \& Reddy 2003)

$$
\Omega_{\mathrm{CFL}}\left(\mu_{\mathrm{q}}, \mu_{\mathrm{e}}\right)=\Omega_{\mathrm{quarks}}\left(\mu_{\mathrm{q}}\right)+\Omega_{\mathrm{GB}}\left(\mu_{\mathrm{q}}, \mu_{\mathrm{e}}\right)+\Omega_{1}\left(\mu_{\mathrm{e}}\right)
$$

with $\mu_{\mathrm{q}}=\mu_{\mathrm{n}} / 3$ where $\mu_{\mathrm{n}}$ is the neutron chemical potential, and

$$
\begin{aligned}
\Omega_{\text {quarks }}\left(\mu_{\mathrm{q}}\right)= & \frac{6}{\pi^{2}} \int_{0}^{v} p^{2} \mathrm{~d} p\left(p-\mu_{\mathrm{q}}\right) \\
& +\frac{3}{\pi^{2}} \int_{0}^{v} p^{2} \mathrm{~d} p\left(\sqrt{p^{2}+m_{\mathrm{s}}^{2}}-\mu_{\mathrm{q}}\right) \\
& -\frac{3 \Delta^{2} \mu_{\mathrm{q}}^{2}}{\pi^{2}}+\text { Bag }
\end{aligned}
$$

with $m_{\mathrm{u}}=m_{\mathrm{d}}=5 \mathrm{MeV}$,

$$
v=2 \mu_{\mathrm{q}}-\sqrt{\mu_{\mathrm{q}}^{2}+\frac{m_{\mathrm{s}}^{2}}{3}}
$$

where $\Omega_{\mathrm{GB}}\left(\mu_{\mathrm{q}}, \mu_{\mathrm{e}}\right)$ is a contribution from the Goldstone bosons arising due to the chiral symmetry breaking in the CFL phase (Shovkovy et al. 2003; Son \& Stephanov 2000a, 2000b; Buballa \& Oertel 2002; Alford et al. 2001; Alford \& Reddy 2003)

$$
\Omega_{\mathrm{GB}}\left(\mu_{\mathrm{q}}, \mu_{\mathrm{e}}\right)=-\frac{1}{2} f_{\pi}^{2} \mu_{\mathrm{e}}^{2}\left(1-\frac{m_{\pi}^{2}}{\mu_{\mathrm{e}}^{2}}\right)^{2}
$$

where

$$
f_{\pi}^{2}=\frac{(21-8 \ln 2) \mu_{\mathrm{q}}^{2}}{36 \pi^{2}} \quad m_{\pi}^{2}=\frac{3 \Delta^{2}}{\pi^{2} f_{\pi}^{2}} m_{\mathrm{s}}\left(m_{\mathrm{u}}+m_{\mathrm{d}}\right)
$$

$\Omega_{1}\left(\mu_{\mathrm{e}}\right)$ is the negative of Equation (8), and the quark number densities are equal, i.e.,

$$
\rho_{\mathrm{u}}=\rho_{\mathrm{d}}=\rho_{\mathrm{s}}=\frac{v^{3}+2 \Delta^{2} \mu_{\mathrm{q}}}{\pi^{2}}
$$

In the above expressions $\Delta$, the gap parameter, is taken to be $100 \mathrm{MeV}$ (Alford \& Reddy 2003).

The electric charge density carried by the pion condensate is given by

$$
Q_{\mathrm{CFL}}=f_{\pi}^{2} \mu_{\mathrm{e}}\left(1-\frac{m_{\pi}^{4}}{\mu_{\mathrm{e}}^{4}}\right)
$$

For the case of strange stars, charge neutrality has to be enforced. Hence, we take $\mu_{\mathrm{e}}$ in the above expressions so that $Q_{\mathrm{CFL}}$ vanishes. In writing down the thermodynamic potential, we neglect the contribution due to the kaon condensation, which is an effect of order $m_{\mathrm{s}}^{4}$ and hence is small compared with the $\Delta^{2} \mu_{\mathrm{q}}^{2}$ contribution to the thermodynamic potential for $\Delta \sim 100 \mathrm{MeV}$. We note that the CFL phase model is a variant of the MIT bag model in which the quarks are allowed to pair. Hereafter, we refer to the model where pairing is present as the CFL phase model.

\section{Results}

In order to describe the crust of hadronic, hybrid, and strange stars we use the well known EOS calculated in Baym et al. (1971) for very low densities. This is justified because all our codes only run up to subnuclear densities of the order of $0.01 \mathrm{fm}^{-3}$ and the crust can, in principle, bear much lower densities.

Next we investigate the differences arising from different EOS for strange matter. In Figure 1 we plot the EOS obtained from the bag model for three values of the bag constants. One can see a clear discontinuity between the hadronic crust and the inner strange matter. This plot is similar to the one shown in Glendenning \& Weber (1992). The differences arising from different bag values appear only at reasonably large energy densities but do affect the properties of the stars. In particular, the larger the bag value, the larger the gap between the crust and the core of the strange star. Note the units: $1 \mathrm{fm}^{-4}=$ $3.5178 \times 10^{14} \mathrm{~g} \mathrm{~cm}^{-3}=3.1616 \times 10^{35}$ dyne cm$^{-2}$.

In Figure 2, we show the differences in stiffness in the EOS obtained from the bag and the CFL phase models within the range of energy densities where they are used.

In previous studies of hybrid stars containing a core of quark matter (Panda et al. 2004b) it was found that stable stars are sensitive to the Bag value. In a hybrid star with the quark phase described by a CFL phase model, for a given gap constant, $\Delta$, a phase transition to a deconfined CFL phase was found possible only for Bag greater than a critical value. For lower values pure quark matter stars were found. For $\Delta=100 \mathrm{MeV}$ one should have $\mathrm{Bag}^{1 / 4} \geq$ $185 \mathrm{MeV}$. On the other hand, for $\mathrm{Bag}^{1 / 4}=190 \mathrm{MeV}$ it was not possible to obtain stable stars with masses equal to most of known radio-pulsar masses. In the present work we also vary the value of the bag constant to check its influence on the properties of the strange stars.

Given the EOS, the next step is to solve the TolmanOppenheimer-Volkoff equations (Tolman 1939):

$$
\frac{\mathrm{d} P}{\mathrm{~d} r}=-\frac{G}{r} \frac{(\varepsilon+P)\left(M+4 \pi r^{3} P\right)}{(r-2 G M)}
$$




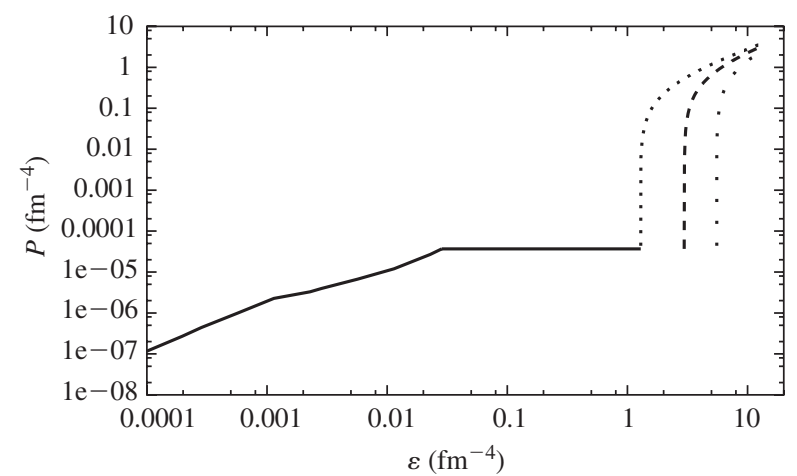

Figure 1 EOS for strange stars obtained with BPS for low densities (solid line) and the bag model for higher densities. From left to right, the curves are for $\mathrm{Bag}^{1 / 4}=145,180$, and $211 \mathrm{MeV}$.

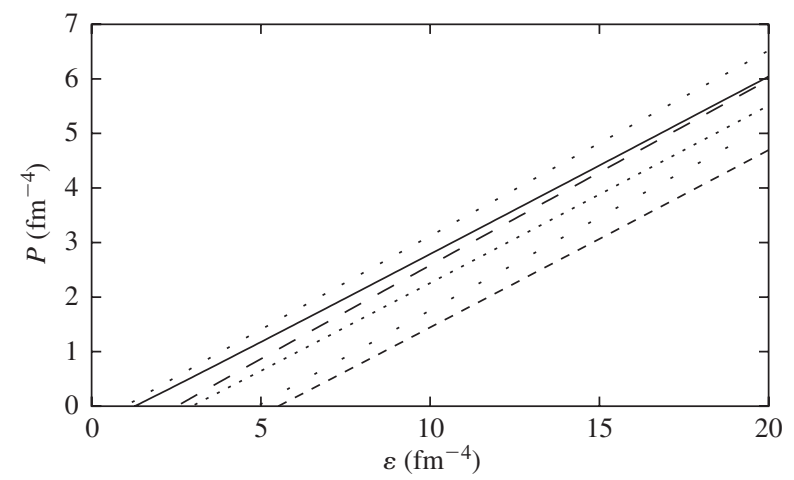

Figure 2 EOS for strange stars obtained with the bag model and the CFL phase model for different values of the bag constant. From left to right the curves are for cfl145, mit145, cfl180, mit180, cfl211, and mit211.

$$
\frac{\mathrm{d} M}{\mathrm{~d} r}=4 \pi r^{2} \varepsilon
$$

with $G$ as the gravitational constant and $M(r)$ as the gravitational mass inside radius $r$. We use units with $c=1$. These equations are integrated from the origin for a given choice of the central energy density, $\varepsilon_{0}$. The value of $r=R$, where the pressure vanishes defines the surface of the star.

In Figure 3 we plot the mass-radius relations of various kinds of stars. In order to understand the role of the crust in each one of them, we display the results of each kind with and without its crust. Once the crust is inserted into the EOS, the radius of the star changes its behavior. One can compare three sets of stars: hadronic, hybrid, and strange stars. In all of them, if no crust is considered, the radius slightly increases and then decreases as the mass decreases. Once the crust is inserted into the EOS, the radius becomes very large beyond a certain critical mass. Actually, the radius can increase up to huge values if densities as low as $10^{-15} \mathrm{fm}^{-3}$ are considered. In order to plot this figure for the hadronic and hybrid stars, we choose the EOS derived in Menezes \& Providência (2004b, 2004c), where the hybrid stars were obtained by the enforcement of Gibbs criterion for phase coexistence. In this case, the EOS is smooth and no discontinuities

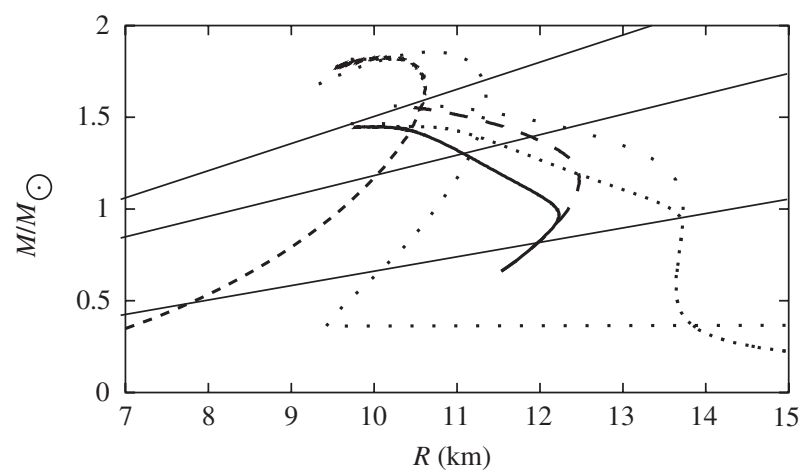

Figure 3 Mass-radius relations for possible three different kinds of neutron stars with and without a crust. From left to right the curves correspond to a strange star for the mit145 model, strange star for mit145 model plus BPS, hybrid star, hybrid star plus BPS, hadronic star, and hadronic star plus BPS. The straight lines correspond to the constraints mentioned in the text.

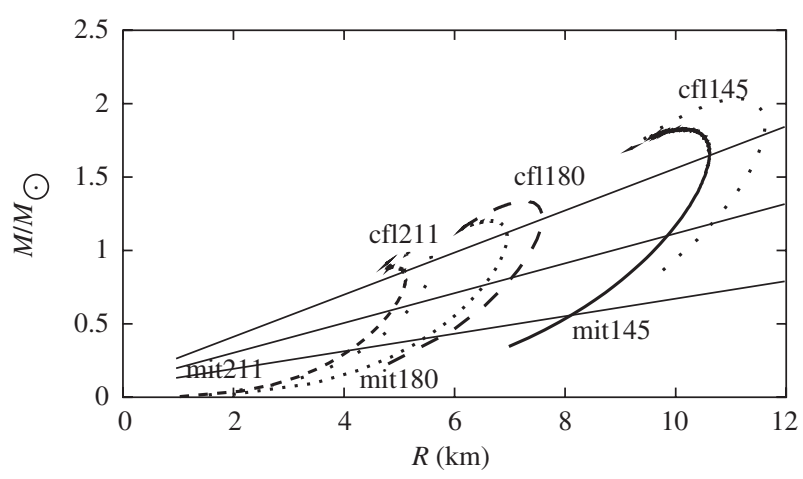

Figure 4 Mass-radius relations for two different models, which can describe strange stars and varying bag constants. The straight lines correspond to the constraints mentioned in the text.

are present. Notice that the results for the hybrid stars seems to interpolate between the results for hadronic and strange stars. Although the general plots of the stars with and without crust look somewhat different, more so for strange stars than for hadronic or hybrid stars, and also the effect of a crust is weaker on the properties of stars near maximum mass.

In Figure 4 we display the mass-radius relations of strange stars. Our aim here is to compare different quark models and for this purpose the inclusion of the crust is not necessary. In both models considered, i.e., MIT bag or CFL phase model, the bag constant was taken as $\mathrm{Bag}^{1 / 4}=145,180$, and $211 \mathrm{MeV}$. In what follows, mit145 always refers to the bag model with a bag constant equal to $\mathrm{Bag}^{1 / 4}=145 \mathrm{MeV}$, cfl211 refers to the CFL phase model with $\mathrm{Bag}^{1 / 4}=211 \mathrm{MeV}$, and so on. Both models show a decrease in the maximum mass with increasing bag constant, which effect is evident from Table 1.

In Table 1, we show the stellar properties for the EOS discussed above, namely, the maximum gravitational and baryonic masses, the central energy density, and the radius of the star with the maximum mass. We do not include the 
Table 1. Hadronic, hybrid, and strange star properties for the EOSs described in the text

\begin{tabular}{llcccccc}
\hline Type & Crust & $\begin{array}{c}M_{\max } \\
\left(M_{\odot}\right)\end{array}$ & $\begin{array}{c}M_{b \max } \\
\left(M_{\odot}\right)\end{array}$ & $\begin{array}{c}R \\
(\mathrm{~km})\end{array}$ & $\begin{array}{c}\varepsilon_{0} \\
\left(\mathrm{fm}^{-4}\right)\end{array}$ & $\begin{array}{c}v_{k} \\
(\mathrm{~Hz})\end{array}$ & $\begin{array}{c}T \\
(\mathrm{~s})\end{array}$ \\
\hline hadronic & No & 1.56 & 1.75 & 10.23 & 7.48 & 805 & 0.0012 \\
hadronic & Yes & 1.56 & 1.74 & 10.59 & 7.67 & - & - \\
hybrid & No & 1.45 & 1.61 & 9.96 & 8.06 & 762 & 0.0013 \\
hybrid & Yes & 1.45 & 1.60 & 10.40 & 8.07 & - & - \\
mit145 & No & 1.82 & 2.33 & 10.15 & 6.59 & 1228 & 0.00081 \\
mit145 & Yes & 1.86 & 2.08 & 10.70 & 6.31 & - & - \\
mit180 & No & 1.20 & 1.25 & 6.66 & 15.20 & 1891 & 0.00053 \\
mit180 & Yes & 1.23 & 1.16 & 7.11 & 12.93 & - & - \\
mit211 & No & 0.88 & 0.80 & 4.91 & 27.65 & 2552 & 0.00039 \\
mit211 & Yes & 0.79 & 0.48 & 5.46 & 12.92 & - & - \\
cfl145 & No & 2.03 & 2.91 & 11.61 & 5.77 & 1136 & 0.00087 \\
cfl145 & Yes & 2.24 & 3.24 & 12.01 & 4.66 & - & - \\
cfl180 & No & 1.34 & 1.52 & 7.33 & 12.04 & 1772 & 0.00056 \\
cfl180 & Yes & 1.40 & 1.50 & 7.75 & 11.76 & - & - \\
cfl211 & No & 0.99 & 0.96 & 5.39 & 22.87 & 2366 & 0.00042 \\
cfl211 & Yes & 0.99 & 0.80 & 5.74 & 17.86 & - & - \\
\hline
\end{tabular}

maximum radius because it can vary depending on how low in density we go with the BPS EOS. With few exceptions, the maximum gravitational mass increases, the maximum baryonic mass decreases, the radius increases, and the central energy density decreases when the crust is included.

We also include a calculation of the Kepler frequency, which determines the spin rate of a neutron star. Based on a simplification of the Kepler frequency for a rigid body, Lattimer \& Prakash (2004) found that the maximum rotation rate (in Hertz) for a star of mass $M$, not close to the maximum mass, and nonrotating radius $R$ is given by

$$
v_{K}=1045 \sqrt{\frac{M}{M_{\odot}}}\left(\frac{10 \mathrm{~km}}{R}\right)^{3 / 2}
$$

We use this prescription to estimate the Kepler frequency and its related period. We calculate frequencies and periods only for stars without the crust. In a strange star, the crust and the core are not connected and one cannot assume rigid body; a detailed discussion on this point is given in Glendenning \& Weber (1992).

As a final example, we consider the EOS for the MIT bag model for $T=10$ and $T=15 \mathrm{MeV}$, which covers the highest temperatures, $\sim 10^{11} \mathrm{~K}$, with $1 \mathrm{MeV}=$ $1.1605 \times 10^{10} \mathrm{~K}$, considered for the surface of a bare strange star (Aksenov et al. 2004). In Table 2 we display the strange star properties for these temperatures and in Figure 5 the corresponding mass radius curves. One sees that for the bag value of $\mathrm{Bag}^{1 / 4}=180 \mathrm{MeV}$, the $T=10$ and $T=15 \mathrm{MeV}$ results are somewhat similar, and for $\mathrm{Bag}^{1 / 4}=211 \mathrm{MeV}$ the $T=0$ and $T=10 \mathrm{MeV}$ are essentially indistinguishable; our codes do not have enough precision to distinguish temperatures so close to each other.
Table 2. Hot strange star properties for the EOSs described in the text

\begin{tabular}{lccccc}
\hline Type & $\begin{array}{c}T \\
(\mathrm{MeV})\end{array}$ & $\begin{array}{c}M_{\max } \\
\left(M_{\odot}\right)\end{array}$ & $\begin{array}{c}M_{b \max } \\
\left(M_{\odot}\right)\end{array}$ & $\begin{array}{c}R \\
(\mathrm{~km})\end{array}$ & $\begin{array}{c}\varepsilon_{0} \\
\left(\mathrm{fm}^{-4}\right)\end{array}$ \\
\hline mit180 & 0 & 1.20 & 1.25 & 6.66 & 15.20 \\
mit180 & 10 & 1.23 & 1.28 & 6.81 & 13.80 \\
mit180 & 15 & 1.22 & 1.27 & 6.75 & 14.61 \\
mit211 & 0 & 0.88 & 0.80 & 4.91 & 27.65 \\
mit211 & 10 & 0.89 & 0.80 & 4.91 & 27.31 \\
mit211 & 15 & 0.90 & 0.81 & 4.98 & 26.96 \\
\hline
\end{tabular}

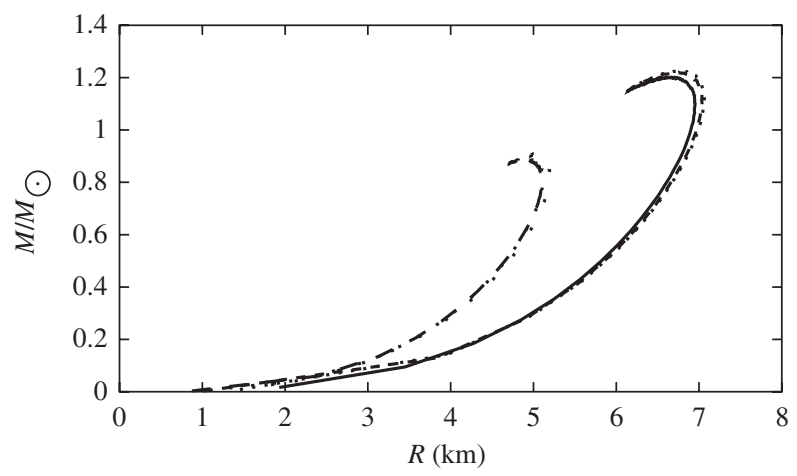

Figure 5 Mass-radius relations obtained with the bag model at three different temperatures and two bag constants. The first set of curves, on the left corresponds to mit211 at $T=0,10$ and $15 \mathrm{MeV}$ and the second to mit180 for the same temperatures.

\section{Discussion and Conclusions}

The measurement of the gravitational redshift of spectral lines produced in a neutron star photosphere can provide a direct constraint on the mass-to-radius ratio. A redshift of 0.35 from three different transitions of the spectra of the X-ray binary EXO0748-676 was obtained 
in Cottam et al. (2002). This redshift corresponds to $M / R=0.15 M_{\odot} \mathrm{km}^{-1}$. Another constraint on the massto-radius ratio is from the observation of two absorption features in the source spectrum of the 1E 1207.4 5209 neutron star, which gives $M / R=0.069 M_{\odot} \mathrm{km}^{-1}$ to $M / R=0.115 M_{\odot} \mathrm{km}^{-1}$ (Sanwal et al. 2002). In this second case, however, the interpretation of the absorption features as atomic transition lines is controversial. Bignami et al. (2003) and Xu et al. (2003) claim that the aborption features are cyclotron line, which imply no obvious constraint on the redshift. In Figures 3 and 4 we add the lines corresponding to these constraints. All the models analyzed in this paper are consistent with the constraints. While the hadronic and hybrid equations of state are not consistent with the complete set of constraints, the strange stars, either crusted or bare, are consistent with all constraints. A similar conclusion concerning strange stars was reached by $\mathrm{Xu}(2003 \mathrm{~b})$.

From Table 1 it follows that strange stars can rotate much faster than either hybrid or hadronic stars, and can have periods as short as 0.4 to $0.8 \mathrm{~ms}$. For comparison, the highest observed spin rate from pulsar PSR B1937+21 is $641 \mathrm{~Hz}$ (Ashworth et al. 1983), and this value is consistent with the pulsar being an ordinary neutron star. According to Glendenning (2000), if a period less that $1 \mathrm{~ms}$ were found, this would be strong evidence that the star must be bound more tightly than by gravity alone, implying a strange star. However, whereas extreme rotation speed can rule out a neutron star, actually, as pointed out in Alcock et al. (1986), in other cases it is difficult to distinguish a strange star from a neutron star. From Table 2 one can conclude that, although strange stars are thought of as hot objects, characterized by super-Eddington luminosities, it is reasonable to neglect the temperature (and to assume $T=0$ ) in a detailed investigation of quark matter.

The possible existence of pentaquarks, that is four quarks and one antiquark confined inside an exotic baryon, has been discussed in the literature (Golowich 1971; Stancu 2005). This hypothesis was confirmed in 2002, when a narrow baryon of strangeness $S=+1$ and mass equal to $1530 \mathrm{MeV}$ was detected (Nakano et al. 2003). The effective mass of a nucleon at nuclear saturation density is about $30 \%$ less than the mass of a free nucleon and a pentaquark is stable only if its effective mass in the medium decreases more than the nucleon effective mass, a condition which is probably not satisfied. However, if it is satisfied, it may also be a possibility for the quark matter in strange stars. As stated in the introduction, the Nambu-Jona-Lasinio (Nambu \& Jona-Lasinio 1961a, $1961 b)$ is also a much richer model than the models used in the present work and it should also be considered in the description of strange matter. These two possibilities are currently under investigation.

\section{Acknowledgments}

This work was partially supported by Capes (Brazil) under process BEX 1681/04-4. D.P.M. would like to thank the friendly atmosphere at the Research Centre for Theoretical Astrophysics in the Sydney University, where this work was done and Dr Constança Providência for the careful reading of the manuscript and useful comments.

\section{References}

Aksenov, A. G., Milgrom, M., \& Usov, V. V. 2004, ApJ, 609, 363

Alcock, C., Farhi, E., \& Olinto, A. 1986, ApJ, 310, 261

Alford, M. G. 2001, ARNPS, 51, 131

Alford, M., Rajagopal, K., Reddy, S., \& Wilezeck, F. 2001, PhRvD, 64, 074017

Alford, M., \& Reddy, S. 2003, PhRvD, 64, 074024

Ashworth, M., Lyne, A. G., \& Smith, F. G. 1983, Natur, 301, 313

Baym, G., Pethick, C., \& Sutherland, P. 1971, ApJ, 170, 299

Bignami, G. F., Caraveo, P. A., De Luca, A., \& Mereghetti, S. 2003, Natur, 423, 725

Bodmer, A. R. 1971, PhRvD, 4, 1601

Buballa, M., \& Oertel, M. 2002, NuPhA, 703, 770

Chodos, A., Jaffe, R. L., Johnson, K., Thorne, C. B., \& Weisskopf, V. F. 1974, PhRvD, 9, 3471

Cottam, J., Paerels, F., \& Mendez, M. 2002, Natur, 420, 51

Espíndola, A. L., \& Menezes, D. P. 2002, PhRvC, 65, 045803

Glendenning, N. K. 2000, Compact Stars (New York: Springer)

Glendenning, N. K., \& Weber, F. 1992, ApJ, 400, 647

Golowich, E. 1971, PhRvD, 4, 262

Ivanenko, D., \& Kurdgelaidze, D. F. 1969, NCimL, 2, 13

Lattimer, J. M., \& Prakash, M. 2004, Sci, 304, 536

Menezes, D. P., \& Providência, C. 2003, PhRvC, 68, 035804

Menezes, D. P., \& Providência, C. 2004a, PhRvC, 69, 045801

Menezes, D. P., \& Providência, C. 2004b, PhRvC, 70, 058801

Menezes, D. P., \& Providência, C. 2004c, Braz. J. Phys., 34, 724

Nakano, T., et al. (LEPS collaboration) 2003, PhRvL, 91, 012002

Nambu, Y., \& Jona-Lasinio, G. 1961a, PhRv, 122, 345

Nambu, Y., \& Jona-Lasinio, G. 1961b, PhRv, 124, 246

Oppenheimer, J. R., \& Volkoff, G. M. 1939, PhRv, 55, 374

Panda, P. K., Menezes, D. P., \& Providência, C. 2004a, PhRvC, 69, 058801

Panda, P. K., Menezes, D. P., \& Providência, C. 2004b, PhRvC, 69, 025207

Prakash, M., Bombaci, I., Prakash, M., Ellis, P. J., Lattimer, J. M., \& Knorren, R. 1997, PhR, 280, 1

Santos, A. M. S., \& Menezes, D. P. 2004, PhRvC, 69, 045803

Sanwal, D., Pavlov, G. G., Zavlin, V. E., \& Teter, M. A. 2002, ApJL, 574,61

Shovkovy, I., Hanauske, M., \& Huang, M. 2003, PhRvD, 67, 103004

Son, D., \& Stephanov, M. 2000a, PhRvD, 61, 074012

Son, D., \& Stephanov, M. 2000b, PhRvD, 62, 059902

Stancu, F. 2005, IJMPA, 20, 250L

Tolman, R. C. 1939, PhRv, 55, 364

Usov, V. V. 1998, PhRvL, 80, 230

Usov, V. V. 2001, ApJL, 550, 179

Witten, E. 1984, PhRvD, 30, 272

Xu, R. X. 2003a, Strange Quark Stars - A Review, in: High Energy Processes and Phenomena in Astrophysics, Proceedings of IAU Symp. 214, eds. V. Trimble, \& Z. Wang, pp. 191-198

Xu, R. X. 2003b, ChJAA, 3, 33

Xu, R. X., Wang, H. G., \& Qiao, G. J. 2003, ChPhL, 20, 314 